Results Echocardiographic global longitudinal strain (GLS) and extracellular volume (ECV) measured by CMR were the only variables able to independently stratify between the three groups of patients. ECV was the best technique for differentiation between hypertensive heart disease and HFPEF (AUC 0.88 ; GLS AUC 0.78, $\mathrm{p}<0.001$ for both). Using ECV, an optimal cut-off of $31.2 \%$ gave $100 \%$ sensitivity and $75 \%$ specificity. ECV was significantly higher and GLS was significantly reduced in subjects with reduced exercise capacity (lower peak $\mathrm{VO} 2$ and higher VE/VCO2).

Conclusions Both GLS and ECV are able to independently discriminate between hypertensive heart disease and HFpEF and identify patients with prognostically significant functional limitation. ECV is the best diagnostic discriminatory marker of HFpEF and could be used as a surrogate end-point for therapeutic studies.

\section{DEMONSTRATION OF CARDIAC AL AMYLOIDOSIS REGRESSION AFTER SUCCESFUL CHEMOTHERAPY. A CMR STUDY}

${ }^{1}$ Ana Martinez-Naharro, ${ }^{2,3}$ Amna Abdel-Gadir, ${ }^{2,3}$ Thomas A Treibel, ${ }^{1}$ Giulia Zumbo, ${ }^{1}$ Daniel S Knight, 1,3Stefania Rosmini, ${ }^{1}$ RN Thirusha Lane, 'Shameem Mahmood, ${ }^{1}$ Sajitha Sachchithanantham, ${ }^{1}$ Carol J Whelan, ${ }^{1}$ Helen J Lachmann ${ }^{1}$ Ashutosh D Wechalekar ${ }^{4}$ Peter Kellman ${ }^{4} J u l i a n$ D Gillmore, ${ }^{2,3}$ James C Moon, ${ }^{1}$ Philip N Hawkins, ${ }^{1}$ Marianna Fontana. ${ }^{1}$ National Amyloidosis Centre, University College London, Royal Free Hospital, London, UK; ${ }^{2}$ Institute of Cardiovascular Science, University College London, London, UK; ${ }^{3}$ Barts Heart Centre, West Smithfield, London, UK; ${ }^{4}$ National Heart, Lung and Blood Institute, National Institutes of Health, Bethesda, Maryland, USA

\subsection{6/heartjnl-2017-311399.8}

Background Cardiac involvement in immunoglobulin light chain (AL) amyloidosis is the major determinant of survival; Cardiac response to chemotherapy is conventionally assessed by serum brain natriuretic peptide (NT-proBNP) and echocardiography, but neither quantify amyloid burden. The aim of this study was to evaluate cardiac AL amyloid serially using cardiovascular MR (CMR) including extracellular volume measurement (ECV), which is the site of the amyloid deposits. Methods 31 patients with cardiac AL amyloidosis who had chemotherapy were studied serially using ECG, echocardiography, ${ }^{123}$ I-labelled serum amyloid P component (SAP) scintigraphy, NT-proBNP measurements and CMR with T1 mapping and ECV measurements (mean interval $20 \pm 11$ months). Nineteen patients achieved a complete or very good partial haematological response (CR $n=10 ; \operatorname{VGPR} n=9)$. Twelve patients attained a partial response (PR) or no response (NR).

Results At follow-up (mean 20 11 months), the amyloid burden had decreased substantially in 6 of the $10(60 \%)$ attaining a CR, 6 of the $9(67 \%)$ in VGPR and 1 of the $8(13 \%)$ in PR. Changes in the ECV consistent with regression of amyloid were concordant with the changes in native $\mathrm{T} 1$, reduction in amyloid volume and in 5 patients with changes in late gadolinium enhancement pattern (figure 1). Overall there was significant reduction in NT-proBNP concentration, LV mass, left atrial area and improvement in diastolic function in patients whose amyloid burden decreased. Regression of cardiac amyloid by CMR correlated with regression of amyloid in other organs measured by SAP scintigraphy.

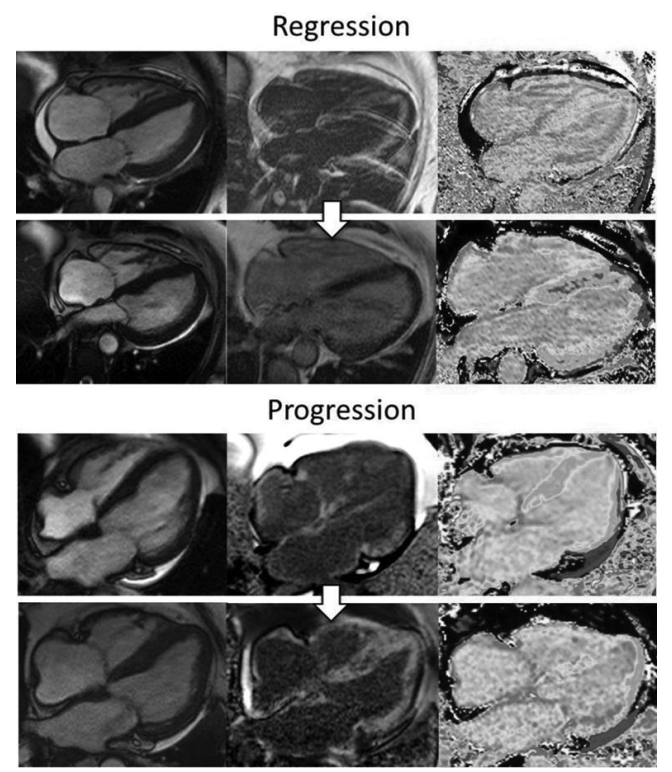

Abstract 008 Figure 1 Top: four chamber SSFP cine images in disatole, corresponding late gadalinium anhancement (LGE) images and ECV mapping before and after chemotherapy in a patient who had regression of amyloid burden after chemotherapy. Bottom: four chamber SSFP cine images in disatole, corresponding LGE images and ECV mapping before and after chemotherapy in a patient who had progression of amyloid burden after chemotherapy.

\section{THE EFFECT OF CARDIAC MAGNETIC RESONANCE ON HUMAN CIRCULATING LEUKOCYTES}

William Critchley, Anna Reid, John Stone, Alexandra Ball, Triin Major, David Clark, Nick Waldron, Christien Fortune, Jakub Lagan, Gavin Lewis, Josephine Naish, Erik Schelbert, Matthias Schmitt, James Fildes, Christopher Miller. University of Manchester; University Hospital of South Manchester NHS Foundation Trust, UK

\subsection{6/heartjnl-2017-311399.9}

Aims Investigators have proposed that cardiovascular magnetic resonance $(\mathrm{CMR})$ should have restrictions similar to those of ionising imaging techniques due to proposed alterations to leukocytes. We aimed to investigate the acute effect of CMR on leukocyte DNA integrity and cell viability in vitro, and in a large cohort of patients in vivo.

Methods and results In vitro study: Peripheral blood mononuclear cells (PBMC) were isolated from healthy volunteers and assessed: 1) immediately following PBMC isolation, 2) after standing on the benchside as a temperature and time control, 3) after a standard CMR scan. Histone H2AX phosphorylation $(\gamma-\mathrm{H} 2 \mathrm{AX})$, an indicator of DNA damage, and leukocyte counts were quantified using flow cytometry. In vivo study: Blood samples were taken from 64 consecutive consenting patients immediately before and after a standard clinical scan. Samples were analysed for $\mathrm{T}$ cell count and $\gamma$-H2AX expression.

CMR scanning was associated with a significant increase in leukocyte $\gamma$-H2AX expression, indicating DNA damage occurs. We also observed a trend towards a significant decrease in absolute leukocyte numbers in vitro following CMR. CMR was not associated with a significant change in $\gamma$-H2AX 